\title{
Slot Allocation for an Integrated Voice/Data TDMA Mobile Radio System with a Finite Population of Buffered Users
}

\author{
Chung-Ju Chang, Member, IEEE, and Chen-Hsiang Wu
}

\begin{abstract}
Slot allocation for voice and data in an integrated TDMA mobile radio system is investigated. In the proposed system, voice traffic is circuit-switched and data traffic is packetswitched using slotted ALOHA for channel access; the data traffic model is practically assumed to have a finite number of users with finite buffer capacity. We apply an equilibrium point analysis (EPA) technique to analyze the data performance and present a heuristic performance criterion to obtain an optimal slot allocation for voice and data in the integrated TDMA mobile radio system.
\end{abstract}

\section{INTRODUCTION}

A TDMA mobile radio system is being expected to provide integrated voice and data services. Since the channel (time slot) capacity shared by these two types of services is limited, how to reasonably allocate a number of time slots to each type of service so as to achieve better balance between voice and data service performance is an important problem. In this paper, we study this problem. To obtain an optimal slot allocation, quantitative performance measures of voice and data must first be available.

In an integrated voice and data TDMA mobile radio system, synchronous transmission (circuit-switched) is suitable for voice service, and asynchronous transmission (packetswitched) is suitable for data service. A random access technique of slotted ALOHA is an appropriate protocol for data service because of the inherent bursty characteristic of data traffic. Performance measures for voice have been given by the $\mathrm{M} / \mathrm{M} / \mathrm{C} / \mathrm{C}$ queueing model [6]; performance analysis of data for ALOHA systems is still under study.

The ALOHA system has been studied for more than 20 years since it was introduced by Abramson in 1970 [1]. Usually, an ALOHA system with an infinite number of users and one unit of buffer capacity is assumed. In recent years, researchers have directed their attention to a buffered ALOHA system in which users have infinite buffer capacity. Sz-

Manuscript received January 18, 1993; revised May 12, 1993. This work was supported by the Telecommunication Labs., Ministry of Transportation and Communications, Republic of China, and the National Science Council, Republic of China, under contracts TL-NSC-81-2102 and the NSC, under 810404-E-009-002.

C.-J. Chang is with the Department of Communication Engineering and Center for Telecommunications Research, National Chiao Tung University; Hsinchu, Taiwan 300, Republic of China.

C.-H. Wu is with the Telecommunication Laboratories, Ministry of Transportation and Communications, Chungli, Taiwan 320, Republic of China.

IEEE Log Number 9213566. pankowski presented a good survey of the performance analysis and system stability of such a system [12]. It is difficult to provide an exact analysis for a general buffered ALOHA system, because the number of states is infinite in the analysis; exact results are only available for some special cases [8], [10], [11]. Sidi and Segall found an explicit expression of mean delay for a system with two identical users in [10] and for a so-called structured priority multiaccess system in [11]. Nain obtained a generating function for a stationary joint queue length distribution in a two-node asymmetrical ALOHA system with the aid of the theory of boundary value problems (Rieman-Hilbert boundary problem) [8]. Results for more than two users are too difficult to derive, so researchers instead turned their attention to developing bounds of performance measures [13], [14], [18] or approximations [3], [4], [9], [15], [16]. Tsybakov and Mikhailov [18] proposed an upper bound, and then Szpankowski [13] extended the results of [18] and found upper and lower bounds for a buffered symmetrical ALOHA system. In [14], Szpankowski further extended his analysis to a buffered asymmetrical ALOHA system. However, the bounds described in [13], [14] are tight only for systems with a small number of users and are unacceptable for systems with more than a moderate number of users.

Saadawi and Ephremides [9] approximated an ALOHA system based on a combination of two Markov chains, in one, referred to as the user Markov chain, describes the state of a user, and the other, referred to as the system Markov chain, describes the state of all users in the system. These two chains are coupled by parameters of boundary condition probabilities and success and collision probabilities, and then an iteration method is used to obtain the final results. This approach is analytically tractable but does not lead to good agreement with simulation results. Ephremides and Zhu achieved better agreement in [3] by expanding the state space of the system so as to track the status of every terminal separately. This excellent approach, however, is not easy to extend to cases with a large number of users, although the authors believed that good agreement still obtains in such cases. Takagi and Kleinrock presented another diffusion approximation in [15]. Fukuda and Tasaka, based on a flow balance concept, introduced an approximate analytical technique termed equilibrium point analysis (EPA) [4], [16]. The EPA method is more analytically tractable than Markovian analysis [3], [5], [9], [17] and more powerful in predicting system stability than S-G (throughout-load) analysis [1]. It has been applied to several 


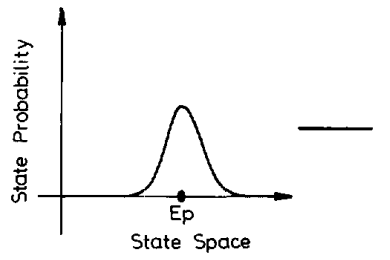

(a) (b)

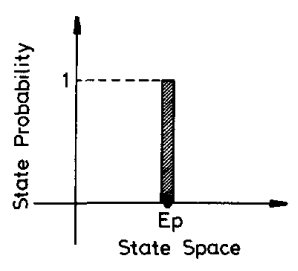

Fig. 1. Approximate probability distribution by the EPA method. (a) Real distribution. (b) Approximate distribution.

cases of ALOHA-type systems. We use this technique to obtain the data performance in this paper.

This paper is organized as follows. EPA is introduced in Section II. In Section III, the equilibrium state of data using the EPA technique is derived, and then the performance measures of the system throughput and the packet delay are obtained. We propose a heuristic performance criterion for determining an optimal slot allocation in Section IV. Finally, concluding remarks are given in Section $V$.

\section{EQUILIBRIUM POINT ANALYSIS}

In 1975, the concept of the equilibrium point in a contentionbased system was first proposed by Kleinrock and Lam [5] and Carleial and Hellman [2]. Carleial and Hellman found that in a statistical sense there exist one or two stable equilibrium points (or states) having high probabilities in an ALOHAtype system; the system either moves around this globally stable equilibrium point, if one stable point exists, or oscillates between these two locally stable equilibrium points, if two stable points exist. Kleinrock and Lam also discovered the interesting fact that the globally stable equilibrium point can be used to make a close approximation of the system behavior. Fukuda and Tasak [4] further identified a flow balance characteristic at the equilbrium point and introduced an analytical technique, called the EPA method, based on this characteristic.

The EPA method approximately assumes that the system is always at an equilibrium point and replaces the actual steadystate probability distribution (illustrated in Fig. 1(a)) by a unit pulse located at the equilibrium point (illustrated in Fig. 1(b)). Also, based on the flow balance property at the equilibrium point, EPA assumes that the expected increment of the number of stations in a mode at an equilibrium point is zero. Note that the mode of a station is characterized by its queue length and its latest transmission history. The procedure for obtaining the equilibrium point (state) is as follows:

Step 1) Define the number of stations in a mode as a state variable and combine all the state variables to be a system state.

Step 2) Calculate the conditional expectation of increment of the number of stations for each node in a slot.

Step 3) Set all expectations in step 2 to zero and solve the simultaneous nonlinear equations to obtain the equilibrium state.

The accuracy of EPA was discussed by Tasaka [16] from the point of view of state probability distribution and a

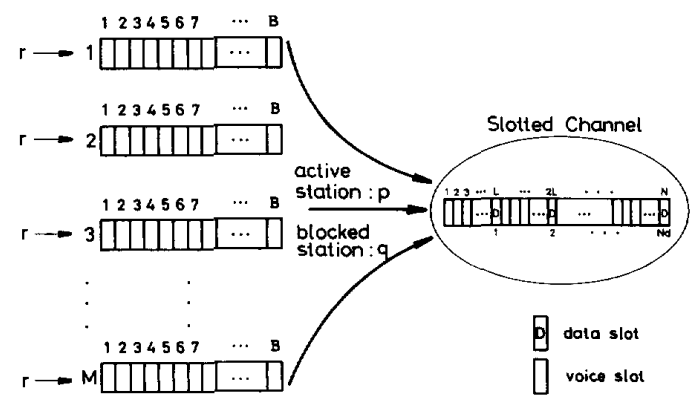

Fig. 2. Model for an integrated voice and data TDMA mobile radio system.

"potential wall." He found excellent agreement between the EPA theoretical result and an exact result when there exists only one globally stable equilibrium point in the system and the state probability distribution is symmetric with respect to the equilibrium point. If there is more than one locally stable equilibrium point (e.g., bistable equilibrium points), EPA selects the one with the worst performance to obtain an approximation. In such a case, though EPA cannot provide a good estimate, we can realize the low bound of the system performance. The reader is referred to [16] for more details on EPA.

\section{ANALYSIS}

Our model for the integrated voice and data TDMA mobile radio system is shown in Fig. 2. The TDMA mobile radio system contains $N$ slots in a frame. The voice service is circuit-switched and the data service is packet-switched using slotted-ALOHA for channel access. A movable boundary multiplexing scheme is adopted, within which the total number of data slots is the sum of nominal data slots and unassigned voice slots. We assume that there are a total of $N_{d}$ slots dedicated to data service and $\left(N-N_{d}\right)$ slots dedicated to voice service at a given time. These $N_{d}$ slots are also assumed to be uniformly distributed over $N$ slots with interslot distance $L=$ $\left\lceil N / N_{d}\right\rceil$ or $\left\lfloor N / N_{d}\right\rfloor$, where $\lceil x\rceil(\lfloor x\rfloor)$ denotes the smallest (largest) integer which is larger (smaller) than $x$. The voice blocking probability can easily be obtained via the Erlang $B$ formula. Thus, we concentrate on the analysis of data service performance in the following.

The system is considered to contain a finite population of $M$ identical data stations, and each station has finite buffer capacity $B$. The status of every station is either active, blocked, or idle, depending on that station's queue length and the history of its latest transmission. A station whose queue is not empty and whose latest transmission succeeded (failed) is called an active (blocked) station and is allowed to transmit the head-of-line packet at the beginning of an upcoming data slot with probability " $p$ " (" $q$ "). The probability " $q$ " is not necessarily equal to " $p$." Also, every station is assumed to have the same traffic load and an independent input in Bernoulli process with mean " $r$ " packets/slot.

\section{A. System State at Equilibrium Point}

Define $\left(N_{1 a}, N_{2 a}, \cdots, N_{B_{a}}, N_{1 b}, N_{2 b}, \cdots, N_{B_{b}}\right)$ as the system state, where $N_{i a}\left(N_{i b}\right)$ is a state variable denoting 
the number of stations belonging to a mode within which each station is active (blocked) and has $i$ packets in its buffer at the beginning of a data slot, $1 \leq i \leq B$. Define $N_{0}$ as the number of idle stations at the beginning of a data slot. Note that $\sum_{i=1}^{B}\left(N_{i a}+N_{i b}\right)+N_{0}=M$. The $\left(N_{1 a}, \cdots, N_{B_{a}}, N_{1 b}, \cdots, N_{B_{b}}\right)$ forms an imbedded Markov chain if observed at the beginning of a data slot. Before the analysis, we also define the following notations and obtain them by

$$
\begin{aligned}
& r_{i} \equiv \operatorname{Pr}(i \text { packets arrive in } L \text { slots }) \\
&=C_{i}^{L} r^{i}(1-r)^{L-i}, \quad \text { for } i=0,1,2, \cdots, L . \\
& S_{a} \equiv \operatorname{Pr}(\text { successful transmission for an } \\
&\quad \text { active station in a data slot }) \\
&=p(1-p)^{\left(\Sigma_{i=1}^{B} N_{i a}-1\right)}(1-q)^{\left(\Sigma_{i=1}^{B} N_{i b}\right)} \\
& S_{b} \equiv \operatorname{Pr}(\text { successful transmission for a blocked } \\
&\quad \text { station in a data slot }) \\
&=q(1-p)^{\left(\Sigma_{i=1}^{B} N_{i a}\right)}(1-q)^{\left(\Sigma_{i=1}^{B} N_{i b}-1\right)} \\
& F_{a} \equiv \operatorname{Pr}(\text { collision probability for an active }\quad \text { station in a data slot }) \\
&= p-S_{a} \\
& F_{b} \equiv \operatorname{Pr}(\text { a blocked station still remains in } \\
&\quad \text { blocked status after a transmission }) \\
&=1-S_{b}
\end{aligned}
$$

where $C_{i}^{L}=L ! / i !(L-i)$ !. Recall that an equilibrium point in the EPA method can be regarded as a state at which the expected increment of the number of stations for each mode is zero. Thus we obtain $2 B$ independent nonlinear equations as follows.

For $N_{i a}$ and $1 \leq i \leq \min (B-1, L)$,

$$
\begin{aligned}
& N_{i a}\left[1-r_{0} \cdot(1-p)-r_{1} S_{a}\right]=N_{0} r_{i}+ \\
& \quad \sum_{x=1}^{i-1}\left\{N_{(i-x) a}\left[r_{x}(1-p)+r_{(x+1)} S_{a}\right]\right\} \\
& \quad+N_{(i+1) a} r_{0} S_{a}+ \\
& \quad \sum_{x=0}^{i} N_{(i+1-x) b} r_{x} S_{b} .
\end{aligned}
$$

For $N_{i a}$ and $L+1 \leq i<B$,

$$
\begin{aligned}
& N_{i a}\left[1-r_{0}(1-p)-r_{1} S_{a}\right]=N_{(i-L) a} r_{L}(1-p)+ \\
& \quad \sum_{x=1}^{L-1}\left\{N_{(i-x) a}\left[r_{x}(1-p)+r_{(x+1)} S_{a}\right]\right\} \\
& \quad+N_{(i+1) a} r_{0} S_{a}+ \\
& \quad \sum_{x=0}^{L} N_{(i+1-x) b} r_{x} S_{b} .
\end{aligned}
$$

For $N_{i a}$ and $i=B$,

1) if $B \leq L$, then

$$
N_{i a}\left(r_{0} S_{a}+F_{a}\right)=N_{0} \sum_{x=B}^{L} r_{x}+
$$

$$
\begin{aligned}
& \sum_{x=1}^{B-1}\left\{N _ { ( B - x ) a } \left[r_{x} \cdot(1-p)\right.\right. \\
& \left.\left.+\sum_{y=x+1}^{L} r_{y}\left(1-p+S_{a}\right)\right]\right\} \\
& +\sum_{x=0}^{B-1}\left[N_{(B-x) b}\left(\sum_{y=x+1}^{L} r_{y} S_{b}\right)\right] ;
\end{aligned}
$$

2) if $L+1 \leq B$, then

$$
\begin{aligned}
N_{i a}\left(r_{0} S_{a}+F_{a}\right)= & N_{(B-L) a} r_{L}(1-p)+ \\
& \sum_{x=1}^{L-1}\left\{N _ { ( i - x ) a } \left[r_{x} \cdot(1-p)\right.\right. \\
& \left.\left.+\sum_{y=x+1}^{L} r_{y}\left(1-p+S_{a}\right)\right]\right\} \\
& +\sum_{x=0}^{L-1}\left[N_{(B-x) b}\left(\sum_{y=x+1}^{L} r_{y} S_{b}\right)\right] .
\end{aligned}
$$

For $N_{i b}$ and $1 \leq i \leq \min (B-1, L)$,

$$
\begin{aligned}
N_{i b}\left(1-r_{0} F_{b}\right)= & \sum_{x=0}^{i-1}\left(N_{(i-x) a} r_{x}\right) F_{a} \\
& +\sum_{x=1}^{i-1}\left(N_{(i-x) b} r_{x}\right) F_{b} .
\end{aligned}
$$

For $N_{i b}$ and $L+1 \leq i<B$

$$
\begin{aligned}
N_{i b}\left(1-r_{0} F_{b}\right)= & \sum_{x=0}^{L}\left(N_{(i-x) a} r_{x}\right) F_{a} \\
& +\sum_{x=1}^{L}\left(N_{(i-x) b} r_{x}\right) F_{b} .
\end{aligned}
$$

For $N_{i b}$ and $i=B$,

$$
\begin{aligned}
N_{i b} S_{b}= & \sum_{x=0}^{\min (B-1, L)}\left[\left(N_{(i-x) a}\left(\sum_{y=x}^{L} r_{y} F_{a}\right)\right]\right. \\
& +\sum_{x=1}^{\min (B-1, L)}\left[N_{(i-x) b}\left(\sum_{y=x}^{L} r_{y} S_{b}\right)\right] .
\end{aligned}
$$

If $B \leq L,(6)$, (7b), and (9) are not included in the set of nonlinear equations; if $L+1 \leq B,(7 \mathrm{a})$ is not included. We did not write the flow equation for $N_{0}$ because it is linearly dependent on the state variables. In each of the above equations, the right-hand side expresses the expected flow moving out of $N_{i y}$, and the left-hand side expresses the expected flow moving into $N_{i y}$, where $1 \leq i \leq B, y=a$ or $b$. Combining (1)-(10) above with constraint $\sum_{i=1}^{B}\left(N_{i a}+N_{i b}\right)+N_{0}=M$, we have $(2 B+5)$ independent nonlinear equations. The nonlinear equations can be numerically solved to obtain the equilibrium point solution $\left(N_{1 a}^{\star}, \cdots, N_{B_{a}}^{\star}, N_{1 b}^{\star}, \cdots, N_{B_{b}}^{\star}\right)$ and $N_{0}^{\star}, S_{a}^{\star}, S_{b}^{\star}, F_{a}^{\star}, F_{b}^{\star}$. Details regarding the numerical solution are presented in the next section. 


\section{B. Performance Measures}

By the assumption of the EPA method that the system is always at an equilibrium point, the system throughout for a given interdistance $L$, denoted by $S(L)$, can be obtained by

$$
S(L)=\sum_{i=1}^{B}\left(N_{i a}^{\star}\right) S_{a}^{\star}+\sum_{i=1}^{B}\left(N_{i b}^{\star}\right) S_{b}^{\star} .
$$

The number of transmission attempts a station takes to complete transmission is a random variable. We denote the random variable of the transmission process for a station by $\Psi_{a}\left(\Psi_{b}\right)$ if the station is in active (blocked) status. Note that the probability of a successful transmission for a station in a data slot is in Bernoulli distribution. If the station is blocked, it has a successful transmission probability $S_{b}^{\star}$ in a data slot at the equilibrium point or a probability $1-S_{b}^{\star}$ of remaining in blocked status. Therefore, the number of data slots that a blocked station takes to complete a transmission follows a geometrical distribution, and the expected value of $\Psi_{b}$, denoted by $E\left(\Psi_{b}\right)$, can be given by

$$
E\left(\Psi_{b}\right)=\frac{1}{S_{b}^{\star}} .
$$

If the station is active, it has a successful transmission probability $S_{a}^{\star}$, a no-transmission probability $1-p$, and a collision probability $p-S_{a}^{\star}\left(F_{a}^{\star}\right)$, in a data slot at the equilibrium point. When an active station has a collision transmission in a data slot, the status of the station is changed to blocked. Fig. 3 is a flow diagram showing the process of $\Psi_{a}$. In the figure, if the packet, initially in an active station, takes only one data slot to complete transmission $\left(\Psi_{a}=1\right)$, the probability is $S_{a}^{\star}$; if the packet takes two data slots to complete transmission $\left(\Psi_{a}=2\right)$, the probabilities are $(1-p) S_{a}^{\star}$ plus $F_{a}^{\star} S_{b}^{\star}$. The probability $(1-p) S_{a}^{\star}$ is the probability that the station has no transmission attempt in the first data slot and has a successful transmission in the second data slot; the probability $F_{a}^{\star} S_{b}^{\star}$ is the probability that the solution has a collision transmission and is changed to blocked station in the first data slot and has a successful transmission in the second data slot; and so on. From this figure, the expected value of $\Psi_{a}$, denoted by $E\left(\Psi_{a}\right)$, can be obtained as follows:

$$
\begin{aligned}
E\left(\Psi_{a}\right)= & \sum_{n=1}^{\infty} n(1-p)^{n-1} S_{a}^{\star}+ \\
& \sum_{k=2}^{\infty}\left[\sum_{n=k}^{\infty} n(1-p)^{k-2} F_{a}^{\star}\left(1-S_{b}^{\star}\right)^{n-k} S_{b}^{\star}\right] \\
= & \frac{S_{a}^{\star}}{p^{2}}+\sum_{k=2}^{\infty}(1-p)^{k-2} F_{a}^{\star}\left[(k-1)+\frac{1}{S_{b}^{\star}}\right] \\
= & \frac{1}{p S_{b}^{\star}}\left(F_{a}^{\star}+S_{b}^{\star}\right) .
\end{aligned}
$$

The average packet delay for a given $L$, denoted by $D(L)$, is the time incurred by an arbitrarily selected acceptable packet from the moment of its arrival until the time its transmission is completed. The average packet delay consists of three components. The first component is the average time between the epoch of the packet's arrival and the instance of

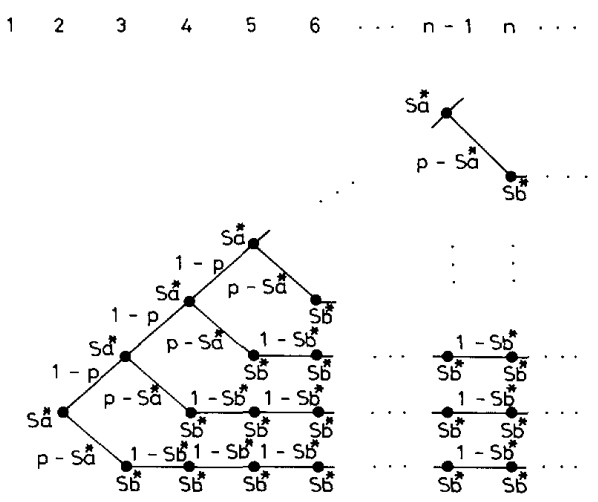

Fig. 3. Flow diagram of the process $\Psi_{a}$.

the beginning of the upcoming data slot. This time can be estimated to be $L / 2$. The second component is the average time between the epoch of the beginning of the next data slot and the time that the selected packet begins to receive service. Since the probability of successful transmission in a data slot for a station follows a Bernoulli distribution, the average service time of the head-of-line packet seen by the selected acceptable packet has the same distribution as the other waiting packets in the queue. If the station is active and there are " $i$ " packets in the station as the selected acceptable packet comes to the station, the second component is equal to $\left[E\left(\Psi_{a}\right) i L\right]$ with probability $N_{i a}^{\star} /\left(M-N_{B_{a}}^{\star}-N_{B_{b}}^{\star}\right)$. If the station is blocked and there are " $i$ " packets in the station, the second component is $\left[E\left(\Psi_{b}\right) L+E\left(\Psi_{a}\right)(i-1) L\right]$ with probability $N_{i b}^{\star} /\left(M-N_{B_{a}}^{\star}-N_{B_{b}}^{\star}\right)$. Note that $1 \leq i \leq B-1$. This third component is the service time of the selected acceptable packet. It is given by $\left[E\left(\Psi_{a}\right)-1\right] \cdot L+1$. Thus, $D(L)$ can be obtained by

$$
\begin{aligned}
D(L)= & \frac{L}{2}+\sum_{i=1}^{B-1}\left\{\frac{N_{i a}^{\star}}{M-N_{B_{a}}^{\star}-N_{B_{b}}^{\star}}\left[E\left(\Psi_{a}\right) i L\right]\right. \\
& \left.+\frac{N_{i b}^{\star}}{M-N_{B_{a}}^{\star}-N_{B_{b}}^{\star}}\left[E\left(\Psi_{b}\right) L+E\left(\Psi_{a}\right)(i-1) L\right]\right\} \\
& +\left[E\left(\Psi_{a}\right)-1\right] \cdot L+1 .
\end{aligned}
$$

For an $N_{d}$-data-slot TDMA mobile radio system, the total of $N$ slots can be considered to contain $n^{+}$data slots with $\left\lceil N / N_{d}\right\rceil$ interdistance with their last neighboring data slot and $n^{-}$data slots with $\left\lfloor N / N_{d}\right\rfloor$ interdistance with their last neighboring data slot, i.e., $N=n^{+}\left\lceil N / N_{d}\right\rceil+n^{-}\left\lfloor N / N_{d}\right\rfloor$, where $n^{+}+n^{-}=N_{d}$ and $n^{+}=N-\left\lfloor N / N_{d}\right\rfloor N_{d}$. Therefore, the mean system throughput and the mean packet delay for a given $N_{d}$, denoted by $\bar{S}\left(N_{d}\right)$ and $\bar{D}\left(N_{d}\right)$, can be obtained by

$$
\begin{gathered}
\bar{S}\left(N_{d}\right)=\frac{n^{-}}{N_{d}} S\left(\left\lfloor N / N_{d}\right\rfloor\right)+\frac{n^{+}}{N_{d}} S\left(\left\lceil N / N_{d}\right\rceil\right) \\
\bar{D}\left(N_{d}\right)=\frac{n^{-}}{N_{d}} D\left(\left\lfloor N / N_{d}\right\rfloor\right)+\frac{n^{+}}{N_{d}} D\left(\left\lceil N / N_{d}\right\rceil\right)
\end{gathered}
$$

where $S(\cdot)$ and $D(\cdot)$ have given in (11) and (14), respectively. 


\section{Numerical ExAMPles AND OPTIMAL Slot AlloCATION}

\section{A. Iteration Method and Simulation}

We shall use Levenberg-Marquardt iterative algorithm in [7] to solve the simultaneous nonlinear equations in (1)-(10). The Intenational Mathematical and Statistical Library (IMSL) has coded this algorithm in its supplied Fortran math library. However, this algorithm requires a good initial guess for iterations. If an unsuitable initial guess is made, the iterative algorithm cannot make good progress and a new guess has to be tried. We here propose a method that can definitely find the solution of a target system with system parameters $\left(p^{\prime}, q^{\prime}, r^{\prime}, M^{\prime}, N_{d}^{\prime}\right)$ if we know a solution of a system with parameters $\left(p, q, r, M, N_{d}\right)$. The method is described as follows. Let $\left(\Delta p, \Delta q, \Delta r, \Delta M, \Delta N_{d}\right)$ denote the parameter difference between a known system and a target system. That is, $\left(\Delta p, \Delta q, \Delta r, \Delta M, \Delta N_{d}\right)=\left(p^{\prime}-p, q^{\prime}-q, r^{\prime}-r, M^{\prime}-\right.$ $\left.M, N_{d}^{\prime}-N_{d}\right)$. It is believed that two systems have quite a similar solution if their corresponding system parametes are close. Thus, in our method, we start from the given solution of the known system and work toward the solution of the target system via $V-1$ temporary transit systems, where $V$ is a suitably large positive integer. We here use $i=0$ to denote the known system, $1 \leq i \leq V-1$ to denote the $i$ th temporary transit system, and $i=V$ to denote the target system. The $i$ th system has the parameters $(p+(i / V) \Delta p, q+(i / V) \Delta q, r+$ $\left.(i / V) \Delta r, M+(i / V) \Delta M, N_{d}+(i / V) \Delta N_{d}\right), 0 \leq i \leq V$. Within the method, we first use the solution of the known system $(i=0)$ as an initial guess for the first transit system $(i=1)$, and then use the solution of the $i$ th system as the initial guess of the $(i+1)$ st system, $i=1, \cdots, V-$ 1. In this way, when implemented on a VAX 8800 super minicomputer, the iterative algorithm in every transit system quickly converges with convergence probability 1 . After $V$ times of temporary transition, we finally obtain the solution for the target system.

We also conduct simulation to help verify the validity of our analysis. We use Little's formula [6] as an indicator to check whether the simulation program should be stopped or not. In other words, we just check whether the mean queue length is equal to the effective arrival rate multiplied by the mean packet delay for a station. Note that the effective arrival rate for a station is the throughput of each individual station $S(L) / M$. For the case of small $N_{d}\left(N_{d}<4\right)$, the simulation stops after about 5000 times of statistics of arriving packets per station; for the case of large $N_{d}$, the simulation stops after about 500 times of statistics of arriving packets per station. The C-language-based simulation program is run on an HP Apollo workstation, which spends $60-600 \mathrm{~min}$ of CPU time for a simulation result.

Figs, 4 and 5 show the mean system throughput and the mean packet delay, respectively, versus the given number of data slots $N_{d}$, where $N=40, M=200, B=10, p=0.008$, and $q=0.0008$. The throughput increases and the delay decreases with the increment of $N_{d}$. The result is intuitively reasonable. Also, the analytical results agree well with the simulation results.

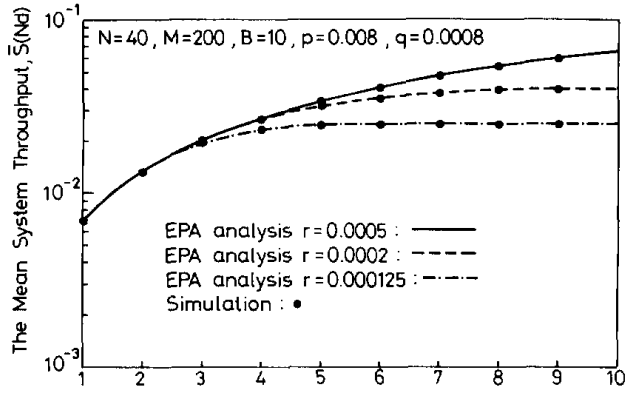

The Number of Data Slots in a Frame, Nd

Fig. 4. Mean system throughput versus the number of data slots $N_{d}$.

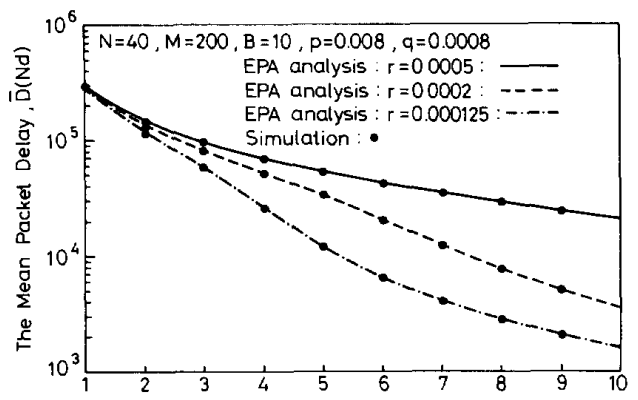

Nd : Number of Data Slots in a Frame

Fig. 5. Mean packet delay versus the number of data slots $N_{d}$.

\section{B. Performance Criterion and Optimal Slot Allocation}

To give a convincing performance criterion for determining optimal slot allocation is a challenging task. A reasonable criterion must be fair to both voice and data services and satisfy system requirements as much as possible. Here, we define a heuristic criterion for a given $N_{d}$, denoted by $P_{c}\left(N_{d}\right)$, as follows:

$$
P_{c}\left(N_{d}\right)=x \ln \left(\frac{P_{B_{\max }}}{P_{B\left(N_{d}\right)}}\right)+(1-x) \ln \left(\frac{\bar{D}_{\max }}{\bar{D}\left(N_{d}\right)}\right)
$$

where $P_{B}\left(N_{d}\right)$ is the voice blocking probability given $(N-$ $N_{d}$ ) voice slots and $\bar{D}\left(N_{d}\right)$ is the mean packet delay given $N_{d}$ data slots, in an $N$-slot frame; $P_{B_{\max }}$ and $\bar{D}_{\max }$ denote the system requirements of the maximum tolerable voice blocking probability and data packet delay; and " $x$ " is a weight parameter balancing voice and data performance. The use of natural logarithmic functions in the performance criterion is to deny those allocations that fail to satisfy system requirements (i.e., $P_{B}\left(N_{d}\right)>P_{B_{\max }}$ or $\left.\bar{D}\left(N_{d}\right)>\bar{D}_{\max }\right)$. Note that the natural logarithmic function is negative if the result of the performance measures violates the system requirements. Also, the use of natural logarithmic functions gives a fairer allocation than that obtained without the use of the natural logarithmic function. This statement can be justified by the following example: An allocation with results of $P_{B_{\max }} / P_{B}\left(N_{d}\right)=20$ and $\bar{D}_{\max } / \bar{D}\left(N_{d}\right)=20$ should be fairer than an allocation with results of $P_{B_{\max }} / P_{B}\left(N_{d}\right)=39$ and $\bar{D}_{\max } / \bar{D}\left(N_{d}\right)=1$. By our performance criterion with $x=0.5$, the former 


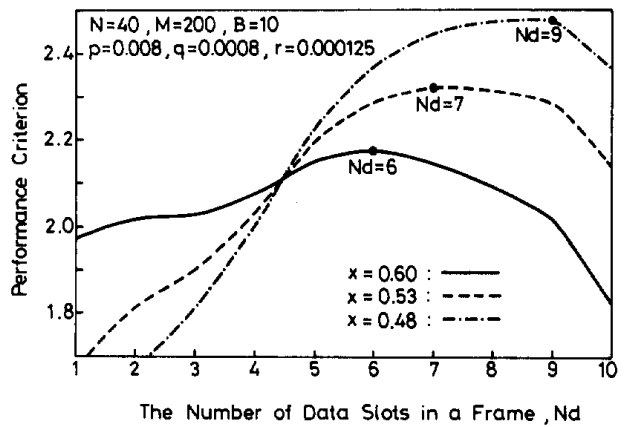

Fig. 6. Performance criterion versus $N_{d}$ for cases with $x=0.60,0.53$, and 0.48 .

allocation obtains a larger value of $P_{c}\left(N_{d}\right)$ than the latter allocation.

Fig. 6 shows the performance criterion versus $N_{d}$, where $N=40, M=200, B=10, p=0.008, q=0.0008$, and the ratio of the voice arrival rate to the service rate of a call in each station is 27 , for cases with $x=0.60,0.53$, and $0.48 . P_{B_{\max }}$ and $\bar{D}_{\max }$ are chosen to be $5 \%$ and $2.25 \times 10^{5}$ (slots), respectively. With the proposed heuristic performance criterion, there exists an optimal number $N_{d}^{\star}$ of data slot allocation of the system, and $N_{d}^{\star}$ is 6,7 , and 9 for the cases where $x=0.60,0.53$, and 0.48 , respectively. From the figure, it is also found that the larger the weight parameter $x$ is (the less weight the system puts on the data performance), the smaller the optimal number of data slots $N_{d}^{\star}$ becomes (the fewer data slots the system provides). This result is obvious.

\section{CONCLUSION}

Optimal slot allocation is an important issue in the design of an integrated voice and data TDMA mobile radio system. In this paper we present a realistic data model by assuming a finite population of finite buffered users for the TDMA system; we use the EPA method to obtain the data performance measures; and we propose a heuristic performance criterion for determining an optimal slot allocation. The numerical examples shown demonstrate that there is an optimal slot number allocated for data for a given traffic load (given $r, p$, and $q$ ) such that the performance criterion is maximized.

\section{REFERENCES}

[1] N. Abramson, "The ALOHA system: Another alternative for computer communications," in Fall Joint Comput. Conf., AFIPS Conf. Proc., vol. 37, 1970, pp. 281-285.

[2] A. B. Carleial and M. E. Hellman, "Bistable behavior of ALOHA-type systems," IEEE Trans. Commun., vol. COM-23, pp. 401-410, Apr. 1975.

[3] A. Ephremides and R. Z. Zhu, "Delay analysis of interacting queues with an approximate model," IEEE Trans. Commun., vol. COM-35. pp. 194-201, Feb. 1987

[4] A. Fukuda and S. Tasaka, "The equilibrium point analysis: $A$ unified analytic tool for packet broadcast networks," in IEEE GLOBECOM, San Diego, CA, 1983, pp. 1133-1140.

[5] L. Kleinrock and S. S. Lam, "Packet switching in a multiaccess broadcast channel: Performance evaluation," IEEE Trans. Commun. vol. COM-23, pp. 410-423, Apr. 1975.

[6] L. Kleinrock, Queueing Systems, Vol. 1: Theory. New York: Wiley, 1975.
[7] More, Jorge, B. Garbow, and K. Hillstrom, "User Guide for MINPACK 1," Argonne National Labs., Argonne, IL, Rep. ANL-80-74, 1980.

[8] P. H. Nain, "Analysis of two-node ALOHA-Network with infinite capacity buffers," IN Int. Sem. Comput. Networking, Perform. Eval. Tokyo, Japan, 1985, pp. 2.2.1-2.2.16.

[9] T. N. Saadawi and A. Ephremides, "Analysis, stability, and optimization of slotted ALOHA with a finite number of buffered users," IEEE Trans. Automat. Contr., vol. AC-26, pp. 680-689, June 1981.

[10] M. Sidi and A. Segall, "Two interfering queues in packet-radio networks," IEEE Trans. Commun, vol COM-31, pp. 123-129, Jan 1983.

[11] "Structured priority queueing systems with applications to packet-radio networks," Perform. Eval., vol. 3, pp. 265-276, 1983.

[12] W. Szpankowski, "Stability, bound and approximation in asymmetric buffered ALOHA-type system," in IEEE Conf. Decision and Control 1986, pp. 1722-1727.

[13] Int. Symp. Perform. Comput. Commun. Syst., Zurich, Switzerland, 1984, pp. 349-366.

[14] "Bounds for queue lengths in a contention packet broadcas system," IEEE Trans. Commun., vol. COM-34, pp. 1132-1140, Nov. 1986.

[15] H. Takagi and L. Kleinrock, "Diffusion process approximation for the queueing delay in contention packet b roadcasting system," in Int. Symp. Perform. Comput. Commun. Syst., Zurich, Switzerland, 1984, pp. 111-126.

[16] S. Tasaka, "Dynamic behavior of CSMA-CD system with a finite population of buffered users," IEEE Trans. Commun., vol. COM-34, pp. 576-586, June 1986

[17] F. A. Tobagi and L. Kleinrock, "Packet switching in radio channel: Part IV: Stability considerations and dynamic control in carrier sense multiple access," IEEE Trans. Commun., vol. COM-25, pp. 1103-1119, Oct. 1977.

[18] B. Tsybakov and M. Mikhailov, "Ergodicity of slotted ALOHA system," Problems Information, Transmission, vol. 15, pp. 73-87, 1979.

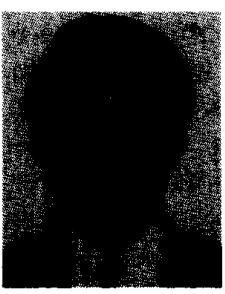

Chung-Ju Chang (S'81-M'85) received the B.E. and M.E. degrees in electronics engineering from National Chiao Tung University, Hsinchu, Taiwan, in 1972 and 1976, respectively, and Ph.D degree in electrical engineering from National Taiwan University in 1985 .

From 1976 to 1988 he was with Telecommunication Laboratories, Directorate General of Telecommunications, Ministry of Communications, Republic of China, as a Design Engineer, Supervisor, Project Manager, and then Division Director. There, he was involved in designing digital switching system, ISDN user-network interface, and ISDN service and technology trials. In the meantime, he also acted as a Science and Technical Advisor for Ministry of Communications from 1987 to 1989 . In August 1988, he joined the faculty of the Department of Communication Engineering and Center for Telecommunications Research National Chiao Tung University, where he is currently professor. His research interests include performance analysis, integrated broadband networks, and mobile radio networks.

Dr. Chang is a member of the Chinese Institute of Engineers (CIE).

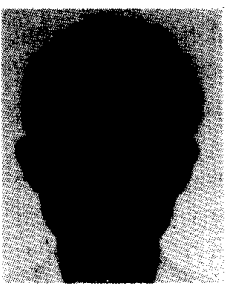

Chen-Shiang Wu was born in Taiwan on November 3, 1967. He received the B.S. degree in electronics engineering from Catholic Fu Jen University, and the M.S. degree in communication engineering from National Chiao Tung University in 1990 and 1992 , respectively.

He is now with the Telecommunication Laboratories, Directorate General of Telecommunications, Ministry of Communications, Republic of China, as a design engineer. There, he is involved in digital subscriber loop technologies. His research interests include mobile radio communication and integrated services digital network. 